\title{
Franquicias: Oportunidad de Negocios Para la Competitividad de Restaurantes y Comidas Rápidas
} en Cúcuta*

\author{
Franchises: business opportunity for fast food restaurants competitiveness in Cucuta \\ Marlly Karina Arenas Torrado** \\ Universidad Simón Bolívar. Cucutá-Colombia
}

Recibido: Febrero 27 de 2017 Aceptado: Junio 15 de $2017 \quad$ Aceptado: Julio 02 de 2017

\section{Resumen}

El objetivo del presente artículo es destacar el modelo de franquicias para restaurantes en el mercado de Cúcuta como estrategia de competitividad y expansión en un mercado amenzado por los conflictos fronterizos; basado en anteriores investigaciones se permitieron los análisis con perspectiva cualitativa mediante investigación documental, así mismo, examina la franquicia como estrategia, sus objetivos relación de franquiciadorfranquiciado, casos de franquicias de comidas rápidas a nivel internacional, nacional y local, resultados que evidencian atractivos prospectos de negocios; es así que el artículo rasalta que el modelo de franquicia para comidas rápidas es una oportunidad de negocios considerando los propectos de mercado evidenciados dado los cambios en los estilos de vida, el crecimiento de la población, los horarios laborales y la mayor participación de la mujer en el mercado laboral, inciden en la demanda de comidas rápidas, fenómeno que en Cúcuta es una realidad, además, además, se aplicó la Investigación Descriptiva mediante la aplicación de un cuestionario a una muestra de la población de 877 restaurantes locales; resalta como conclusión que los hallazgos encontrados constituyen temas de interés para emprendedores, incubadoras de empresas, gremios como Cotelco y población académica, especialmente el estudio de mercado y las recomendaciones en materia de marcas.

Palabras Clave: Competitividad, estrategia, franquicia, prospecto, regalias.

\begin{abstract}
This article aims to highlight restaurants' franchising approach in Cucuta's market, as a competitiveness strategy and market expansion threatened by border conflicts: based on previous researches, analysis with a qualitative point of view, through a documentary research was allowed. Likewise, it examines franchising as a strategy, its franchisefranchisee relationship objectives, cases such as national, international and local fast food restaurants franchising, results that show attractive business prospects; This, this paper highlight that fast food franchise model is a business opportunity considering the market prospects due to lifestyles changes, population growth, work schedules and greater participation of women in the labor market, affecting the demand for fast food in Cucuta; in addition, A descriptive research was applied through a questionnaire; 877 local restaurants were sampled; As a conclusion, the research findings show that this is an important topic for entrepreneurs, business incubators, restaurants unions such as Cotelco and marketing academic population and recommendation on trademarks.
\end{abstract}

Key Words: Competitiveness, franchise, prospect, royalties, strategy.

\section{Este artículo se puede referenciar}

Arenas-Torrado, M. (2017). Franquicias: Oportunidad de Negocios Para la Competitividad de Restaurantes y Comidas Rápidas en Cúcuta. En Desarrollo Gerencial Revista de la Facultad de Ciencias Económicas Administrativas y Contables de la Universidad Simón Bolívar-Colombia, 9(2), 158-173.

\footnotetext{
* Artículo resultado de la investigación titulada Diagnóstico Estratégico y evaluación de acciones frente al potencial de franquicias de Restaurantes en el mercado de Cúcuta, realizado en la Universidad Francisco de Paula Santander, División de Posgrados, Programa Maestría en Gerencia de Empresas

** Universidad Simón Bolívar. Email: m.arenast@unisimonbolivar.edu.co.

Desarrollo Gerencial, 9 (2) Pp. 158-173. Julio-Diciembre 2017. ISSN: 2145-5147 (On Line). Universidad Simón Bolívar. Barranquilla-Colombia. Contactos: desarrollogerencial@unisimonbolivar.edu.co
} 


\section{1.- Introducción}

La evidente crisis social y económica que atraviesa San José de Cúcuta a causa principalmente de la devaluación sistemática del bolívar y al deterioro de las relaciones comerciales con Venezuela, están amenazando a la economía fronteriza e incidiendo en la calidad de vida de las familias, afectadas por la falta de oportunidades de trabajo.

De acuerdo a estas consideraciones, el panorama al corto y mediano plazo no es halagador a pesar de las diversas oportunidades que ofrecen los mercados nacionales e internacionales mediante el aprovechamiento de modelos de expansión como son las relaciones de franquicia con empresas exitosas que brindan ventajas como las de ampliar cobertura de mercado, fortalecimiento de su marca y minimización del riesgo dentro de un proceso de conocimiento y asesoría, como respuestas al siguiente interrogante: ¿Qué acciones se deben emprender para fomentar la modalidad de franquicias en los restaurantes de Cúcuta para ser más competitivos según su situación actual?

Cabe señalar que la modalidad de franquicia involucra actores y partes claves como el franquiciador o persona natural o jurídica que ha desarrollado un negocio relacionado con un producto o servicio y espera expandirse en un mercado específico a través de inversionistas o franquiciados que adquieren el derecho a operar aprovechando la experiencia y la marca ofrecida; modalidad que fortalece la competitividad concepto que equivale a "participar en el mercado con productos, bienes o servicios diferenciados; esta diferenciación puede estar dada por atributos como especificaciones de diseño y materiales, innovación y desarrollo, atención a las necesidades de los consumidores, costo y calidad, entre otros." (Gómez, 2012, p.59). Aunado también al proceso de emprendimiento como elemento de inclusión competitiva, con lo exponen Duran, Fuenmayor, Cárdenas y Hernández (2016) y Fuenmayor, Parra \& Duran (2015)

En atención a lo expuesto, se desarrolla el presente artículo con el propósito de destacar el modelo de franquicias como una oportunidad para restaurantes y comidas rápidas en el mercado de Cúcuta como una importante estrategia de competitividad para el crecimiento y expansión que permite fortalecer al sector dado los prospectos de negocios percibidos a través de diversas investigaciones realizadas que están referenciadas como fuentes secundarias de información que permitieron los análisis pertinentes dentro de una perspectiva cualitativa a través de una investigación documental para el desarrollo de los objetivos específicos.

En este sentido los objetivos del artículo son analizar el Estado del Arte según antecedentes o experiencias anteriores, compendiar el tema de la franquicia como estrategia a través de sus objetivos, orígenes y resultados en Colombia y determinar las actitudes hacia las franquicias de parte de los restaurantes locales mediante Investigación Descriptiva a través de la aplicación de un cuestionario a una 
muestra de la población de 877 restaurantes locales; estos objetivos específicos delimitan el artículo determinando así su alcance.

Dentro de este marco, surge la justificación teórica fundamentada en teorías y conceptos sobre el enfoque de franquicias con el propósito de familiarizar a la investigadora en el tema objeto del conocimiento, dado que permite "profundizar en uno o varios enfoques teóricos que tratan el problema que se explica, a partir de los cuales espera avanzar en el conocimiento planteado" (Méndez, 2007, p.196); en materia metodológica, la justificación está centrada en la aplicación de investigación documental dentro del método deductivo con criterio cualitativo, procedimiento que se fundamenta en la recolección de datos según fuentes secundarias de información basadas en proyectos, artículos y documentos sobre franquicias y sector de restaurantes, además, mediante Investigación Descriptiva aplicó un cuestionario, instrumento previamente validado por expertos, aplicado a una muestra de la población de 877 restaurantes locales y en materia práctica la justificación se refiere a la contribución del artículo a la toma de decisiones de parte de los empresarios del sector local de restaurantes y comidas rápidas en la iniciación de un nuevo negocio a menor riesgo dentro de un proceso de conocimiento y asesoría que impartirá el franquiciador, al respecto Méndez se refiere al "interés del investigador por acrecentar sus conocimientos, obtener un título académico o, si es el caso, por contribuir a la solución de problemas concretos que afectan a organizaciones empresariales" (Méndez, 2007, p.196).

De acuerdo a estas consideraciones, la incursión en un negocio de franquicia requiere el análisis de variables claves como la capacidad competitiva del franquiciador y franquiciado, la inversión y regalías y los prospectos de mercado o tamaño del potencial del mercado de restaurantes de comidas rápidas en Cúcuta y su Área Metropolitana, variables que a su vez constituyen una limitante para iniciar un negocio de franquicia que debe subsanarse con investigación de mayor nivel de profundidad.

\section{2.- Fundamento Teórico}

El proceso investigativo exige mayor rigurosidad para comprender la realidad estudiada mediante análisis de anteriores investigaciones, estudios y valoraciones sobre el tema objeto del conocimiento como es el caso de las franquicias; en tal sentido, las técnicas de recolección y procesamiento de la información permiten evidenciar el desempeño del fenómeno estudiado en materia de estrategias tendientes a la expansión y crecimiento empresarial a través de modelos de franquicias.

$\mathrm{Al}$ respecto, las valoraciones y análisis de experiencias anteriores sobre el tema de estudio representan el Estado del arte, entendido como una "valoración crítica, expresada en un resumen o reseña de cada trabajo, sobre si es un aporte o no al tema de investigación, y en qué aspecto” (Barriga, 2011, p.236), según lo expuesto, el estado del arte se realiza en forma ordenada para permitir investigaciones posteriores que permitan "indagar qué aspectos han quedado sin profundizar, si existen vacíos sobre 
determinado tema, o posturas que sean rebatibles; evitando, en todo caso, pérdida de tiempo y energía para el investigador, al repetir un trabajo ya explorado a profundidad" (Barriga, 2011, p.236)

Dentro de este orden de ideas, algunas fuentes secundarias de información seleccionadas son analizadas y tenidas en cuenta dado sus aportes más relevantes para el presente artículo como es el documento "Programa de Promoción de Exportación de Servicios, (2004)," de la Comisión de la Promoción de Perú para la exportación y el Turismo, Promperú, documento que señala a la franquicia en Colombia como un negocio en auge, es un valioso antecedente que destaca a la Nación colombiana como la cuarta economía más importante de América Latina, después de Brasil, México y Argentina con un "PIB aproximado de 396 mil millones de dólares.

La distribución por actividad económica fue 13,9\%agricultura, 30,3\% industria y 55,8\% servicios; con un crecimiento proyectado del 4\% para el 2011”, además, señala el documento que el tamaño de su población y su ubicación geográfica estratégica generan oportunidades como es el caso de la modalidad de franquicias que constituyen una alternativa importante de realizar negocio a menor riesgo y con rentabilidad asegurada, indica que " el mercado colombiano aún no está lo suficientemente desarrollado, son 300 redes con 6,242 franquicias, por lo cual aún hay espacio para seguir estableciendo más negocios en este mercado emergente", modalidad que principalmente está más concentrada en ciudades como Bogotá con el 36\% de participación en el total de franquicias, Medellín con el 22\%, Cali con el 11\% y el resto de ciudades con una participación del $31 \%$ del total de franquicias; este artículo también muestra algunos indicadores sobre oferta y demanda en materia de relaciones de franquicia en la que el sector de restaurantes y comidas rápidas tienen una presencia significativa.

Al anterior aporte, se suma el señalado por Schlesinger, María, en su estudio "Percepción del posicionamiento estratégico en el ámbito de las franquicias de comida rápida en España," de la Facultad de Ciencias Económicas y Sociales de la Universidad del Zulia. Venezuela, artículo elaborado en 2008 , cuyo propósito es establecer el posicionamiento estratégico de los establecimientos de comidas rápidas en Valencia, España; destaca algunas teorías de diversos autores como Miles y Snow relacionadas con el posicionamiento estratégico necesarias para categorizar a estas empresas y la tipología de las estrategias que deben tenerse en cuenta, para lo cual desarrolla una investigación a través de un muestreo sistemático a gerentes que manejan franquicias de comidas rápidas, cuyos análisis muestran las tendencias a desarrollar capacidades de marketing y de innovación, lo cual es un importante aporte para el presente proyecto, valioso aporte para el presente artículo que será tenido en cuenta.

Según este orden de ideas y con el objeto de entender el crecimiento empresarial mediante franquicias, Felipe Mosquera Muñoz aporta este importante tema en su artículo "La Franquicia una Estrategia de Crecimiento Empresarial"(2010) de la Revista MBA EAFIT , el cual expone los temas básicos del modelo 
de franquicia, tal como opera en el medio colombiano, lo que constituye una valiosa guía para emprendedores que deseen iniciar una idea productiva así como a empresas legalmente establecidas que tengan dentro de sus planes la expansión en segmentos de mercados de interés, es así que las orientaciones impartidas en el documento representan un aporte que será tenido en cuenta en el artículo y en posteriores investigaciones en esta materia, así mismo el análisis que contiene respecto a la relación franquiciadorfranquiciado franquiciante y también desde la posición del franquiciado, a lo que se suma la identificación de ventajas y desventajas en un contrato de franquicia.

En atención a lo expuesto, cabe destacar un importante aporte para el presente artículo relacionado con el fortalecimiento de los negocios de comidas rápidas basado en el modelo de franquicia, explicado en "KLISTO: Franquicia como una estrategia de expansión" elaborado por María Camila Rodríguez Benítez y Nestor Camilo Mora Vargas de la Universidad Colegio Mayor de Nuestra Señora del Rosario, Facultad de Administración, documento que analiza el crecimiento acelerado del sector de comida rápida a nivel nacional, a nivel latinoamericano y a nivel mundial "debido a los cambios en el día a día de los consumidores, en donde la prioridad es el tiempo y este genera unos acelerados ritmos de vida" (Sirgado \& Lamas, 2011), crecimiento que es demostrado según las cifras que registra y que evidencian una expansión del sector representado en mayor número de establecimientos, se suman a los aportes descritos "los conceptos fundamentales a entender de este tipo de negocio, así como las ventajas que posee y las clases de franquicia que se pueden encontrar"

Complementando los anteriores análisis, un importante aporte aparece en el artículo "El Caso de Perdurabilidad Empresarial de Crepes \& Waffles desde una Perspectiva de Responsabilidad Social Empresarial e Innovación en Valor en Colombia” de Vivian Milena Riaño Mosquera de la Universidad del Rosario, Escuela de Administración, Maestría en Dirección de Empresas (2013), el cual es una guía que aporta como ejemplo de expansión y crecimiento el caso dela multinacional Crepes \& Waffles, con unos antecedentes basados en la capacidad de emprendimiento y "arte en la gestión, con la cual construye un valor agregado diferenciador en la industria gastronómica de Colombia, pues mediante el uso de una estrategia enfocada hacia la Responsabilidad Social Empresarial (RSE)", orientación que le ha generado un alto nivel de satisfacción de parte de los clientes externos e internos ñp que constituye un factor clave de éxito en los propósitos de sostenibilidad en los mercados y nichos de mercados que atiende.

En este orden de ideas, merece la pena destacar el importante papel que desempeñan los inventarios en los negocios de franquicias, reflexión contenida en Vílchez Gil, Alirio "Gestión de inventario. Relación con los proveedores en franquicias de comida rápida" que aparece en la Revista Venezolana de Gerencia (2003) de la Universidad del Zulia Maracaibo, Venezuela, su principal aporte está representado en el criterio sobre la optimización de inventarios para minimizar costos de almacenamiento y maximizar 
rendimientos financieros, "dado que la inversión en inventarios representa un porcentaje elevado dentro de la inversión global de una empresa, la importancia de la administración de dicha inversión aumenta día a día”, es así que el objetivo del documento es analizar la gestión de los inventarios en las franquicias de comida rápida y "su relación con los proveedores, a partir de su evolución y elementos que lo caracterizan dado el éxito de este tipo de negocio."

Finalmente, una experiencia a nivel Cúcuta es el proyecto elaborado por Delgado, Diego, (2009), “Análisis Socio Económico y de Mercado del Sector de Comidas Rápidas en San José de Cúcuta”, según los factores sociales, económicos y de mercado que inciden en su comportamiento, proyecto de Administración de Empresas de la Universidad Francisco de Paula Santander que tiene como objetivos suministrar las bases teóricas y conceptuales sobre los pilares y factores socio económicos y de mercado para la sostenibilidad empresarial; determinar la situación actual del sector de comidas rápidas de San José de Cúcuta frente a la sostenibilidad esperada e identificar las estrategias que las empresas de comidas rápidas deben desarrollar para elevar su nivel de productividad y competitividad, objetivos que se desarrollaron mediante investigación documental y descriptiva a una muestra representativa de 129 Restaurantes de Comidas Rápidas, cuyos resultados determinaron que este sector es vulnerable a pesar de contar con más fortalezas que debilidades y con más oportunidades que amenazas, y por lo tanto no tiene asegurado un crecimiento sostenible, aspectos que como diagnóstico constituyen un valiosos aporte para el proyecto.

\section{1.- Franquicia Como Estrategia}

Los propósitos de expansión empresarial, diversificación de segmentos de mercados y fortalecimiento de marca pueden logarse a través de la modalidad de franquicias, considerado un enfoque novedoso de negocios que apunta hacia la Expansión y Desarrollo de Negocios con minimización de riesgos, Inversiones Estratégicas, Estrategias de Comunicación mediante compra de espacios en los diferentes medios, Estrategia de Marca orientada al posicionamiento en los mercados de interés y Desarrollo de Actividades de Mercadotecnia. A esto Prieto y Villa, (2011) sugieren que deben existir sistemas de mercadeo, de manera que las empresas puedan ser parte del actual avance del mundo, así como parte de las estrategias gerenciales propuestas por Duran, Crissien, Virviesca y García (2017)

Dentro de este orden de ideas, cabe destacar los objetivos de la franquicia como estrategia empresarial, "1) Expandir la empresa en el mercado; 2) Buscar socios; 3) Establecer un sistema de cooperación; 4) Compartir riesgos; 5) Ampliar la capacidad financiera; 6) Aperturar mercados para mejorar el posicionamiento de la empresa a nivel competitivo y 7) Asegurar un lugar en la economía globalizada" (Vílchez, 2003, p.514), \& Prieto, Burgos, García y Rincón (2016) 
En atención a lo expuesto, la modalidad de franquicia en Colombia ha mostrado resultados significativos y por lo tanto es considerada como una valiosa oportunidad para generar valor económico agregado como así lo registra el documento Perfil de mercado de Franquicias en Colombia señalando que "Colombia presenta oportunidades para generar franquicias rentables, situación que se sustenta por el comportamiento de indicadores que considera la oferta y la demanda del sector" (PROMPERÚ, 2004, p.8). Para lograr esto es necesario el desarrollo de competencias gerenciales, propuestas por García, Duran \& Prieto (2017).

Es así que el modelo de franquicia apunta hacia la expansión de mercado, utilizado por empresas exitosas, que desean difundir el mercadeo de sus productos y servicios por medio de terceras empresas o puntos de venta formales e independientes utilizando el nombre o marca registrada junto con las técnicas de mercado o formatos de negocio de la casa matriz o franquiciador, a cambio del pago del reconocimiento de una licencia que implica una inversión inicial para el funcionamiento y el pago de unas regalías aplicadas a las ventas.

Al respecto, es importante conocer los antecedentes de la modalidad de negocios a través de franquicias, teniendo en cuenta que "el modelo de franquicia tiene su origen en Estados Unidos y después del éxito que se experimentó en la época y el boom que tuvo este sistema, comenzó a darse una expansión, la cual se ve reflejada aun en los años dos mil, ya que el sector de franquicias en Estados Unidos está considerado como el mercado de mayor madurez y el más grande del mundo" (Rodríguez y otro, 2012, p.25) Escalente \& García (2010).

Cabe resaltar que la franquicia es una relación en la cual intervienen dos entes principales, el franquiciado quien realiza la inversión y el franquiciante o franquiciador quien ha creado un modelo de negocio para algún producto o servicio, actores que suscriben un contrato de colaboración de franquicia "donde para el logro del objeto se necesita que las partes se asistan entre sí de manera estrecha y permanente50, una de las principales características de la franquicia es la llamada "independencia empresarial" que impregna las relaciones entre franquiciador y franquiciado" (Navas y otro, 2009, p.293) García, Duran, Parra \& Marceles (2017) sostienen que esto se genera como una relación de direccionamiento estratégico.

En consecuencia, la franquicia exige una estrecha relación comercial entre franquiciador y franquiciado que debe reglamentarse a través de un Contrato de franquicia que debe contener los siguientes elementos objetivos esenciales como: "1. Derechos de propiedad intelectual (signos distintivos, nuevas creaciones, derechos de autor y variedades vegetales), 2. Know-How (saber-hacer) o secreto empresarial. 3. Asistencia técnica y contable. 4. Precio" (Quiroga, 2012, p.07). 
Dentro del anterior marco, es evidente que la estrategia empresarial basada en el modelo de franquicias genera altos niveles de competitividad, menores riesgos, rápido posicionamiento y atractivos prospectos de negocios, dado que

"en la actualidad, fenómenos como la globalización y el desarrollo tecnológico se acompañan de procesos importantes de cambio alimentario. Estos cambios se expresan en transformaciones de las costumbres alimentarias con la introducción de alimentos pero, sobre todo, de preparaciones foráneas. La denominada comida rápida es sin duda la variación más importante, introducida principalmente en la dieta de jóvenes y adolescentes, aunque existen variaciones importantes por clase social en cuanto a la utilización de este tipo de comida" (Uribe, 2006, p.229)

Cabe considerar que el contrato de franquicia internacional, como sistema de comercialización, "está en constante expansión y se ha convertido en uno de los contratos de colaboración empresarial más utilizados en el escenario de los negocios internacionales contemporáneos. Franquicias como McDonald's, Burger King, Zara, Body Shop, entre otras, son ejemplos del crecimiento de esta figura en el mundo" (Llain-Arenilla y otra, 2016, p.24)

En este sentido, en Colombia en el 2014 "se abrieron cerca de 5000 restaurantes; en este mismo año se estimó que en Colombia había 580 franquicias de las cuales el 36\% son del sector de comidas"(OCEX, 2016, p.06); al respecto, merece la pena resaltar que "las principales franquicias gastronómicas de Colombia son de origen nacional, cadenas como El Corral, Crepes \&Waffles, Juan Valdez, Oma, Frisby, Kokoriko, PPC, Sandwich Qbano, entre otros, (...) sin embargo, las cadenas extranjeras, como Starbucks, Dunkin’ Donuts, Domino's Pizza, Mc Donald's, Burger King, Oxxo, Subway, KFC, entre otros, son cadenas procedentes de Estados Unidos, México, Argentina, Brasil y España” (OCEX, 2016, p.06)

$\mathrm{Al}$ respecto, es importante señalar que en materia de preferencias de los consumidores y con base en una investigación a los 85 principales establecimientos de venta de comida se "encontró que en el año 2015 las ventas se enfocaron en el segmento de hamburguesas con un $26 \%$, pollo con $21 \%$, sándwiches con $10 \%$, pizza con $8 \%$, parrilla con $6 \%$ y típica (incluye comida nacional e internacional) con $3 \%$ (OCEX, 2016, p.16)

A título ilustrado, es de vital importancia considerar que "con la entrada en vigencia del Tratado de Libre Comercio (TLC) suscrito entre Estados Unidos y Colombia, se espera un incremento de franquicias internacionales entre ambos Estados" (Llain-Arenilla y otra, 2016, p.24)

Ahora bien, es así que los resultados anteriores evidencian oportunidades en el desarrollo de franquicias como una alternativa que el sector de restaurantes de San José de Cúcuta debe aprovechar para contribuir al fortalecimiento de la dinámica social y económica de la localidad, es por ello que se requiere de un proceso investigativo para determinar la situación actual de estos establecimientos, identificar las estrategias y acciones que los empresarios del sector de interés deben tener en cuenta con el propósito de 
lograr una efectiva integración con empresas exitosas a través de las relaciones de franquicia como una opción para la sostenibilidad en el mercado.

\section{3.- Método}

\section{Diseño}

El método de la investigación es deductivo, teniendo en cuenta que del análisis de fenómenos generales se llegaron a conclusiones particulares, es así que el enfoque global de las franquicias permitirá mediante la investigación comprender su aplicabilidad a casos particulares como los restaurantes de Cúcuta, al respecto se explica que

"La deducción es un método de razonamiento que lleva a conclusiones partiendo de lo general, aceptado como válido, hacia aplicaciones particulares. Este método se inicia con el análisis de los postulados, teoremas, leyes, y principios de aplicación universal y, mediante la deducción, el razonamiento y las suposiciones, entre otros, se comprueba su validez para aplicarlos en forma particular" (Ramírez, 1996, p.38)

En este sentido, Méndez señala que el método de investigación se ajusta a un "procedimiento riguroso formulado de una manera lógica que el investigador debe seguir en la adquisición de conocimientos" (Méndez, 2007,p.236), procedimiento que se fundamenta en la recolección de datos según fuentes secundarias de información contenidas en redes de información científicas, herramientas o recursos importantes que permitieron la búsqueda y selección de datos mediante Investigación Documental basada en proyectos, artículos y documentos sobre franquicias y sector de restaurantes, con el propósito de orientar a la investigadora en el tema objeto de estudio, dado que se refiere al "estudio de problemas con el propósito de ampliar y profundizar el conocimiento de su naturaleza, con apoyo, principalmente, en trabajos previos" (Universidad Pedagógica Experimental Libertador, Manual de Trabajos de Grado de Especialización y Maestría y Tesis Doctorales, 2002, p.28); al respecto, cabe resaltar que la investigación permite orientar posteriores investigaciones.

\section{Participantes}

En este orden de ideas, merece la pena señalar que para un mayor nivel de profundidad o segundo nivel de conocimiento para determinar las actitudes hacia las franquicias de parte de los restaurantes locales y el desempeño del sector, se aplicó la Investigación Descriptiva dado que permite "establecer comportamientos concretos (cuántas personas consumen un producto; cuál es su actitud frente a su líder, cuáles son las necesidades de la gente)" (Méndez, 2007 p. 231 mediante la aplicación de un cuestionario o instrumento previamente validado por expertos, diseñado con preguntas dicotómicas y otras cerradas que se aplicaron a una muestra de la población de restaurantes locales o mercado meta definido como "el grupo de elementos que tienen unas características en común y para el cual las conclusiones de la investigación son válidas" (Jany,2000 p. 95), población que está identificada como restaurantes de Cúcuta, 
que de acuerdo a datos suministrados por la Cámara de Comercio de Cúcuta se cuantifican en 877 establecimientos (Delgado, 2009, p.25), población analizada a través de una muestra de 87 restaurantes mediante el tipo de muestreo probabilístico aleatorio simple, muestra que se seleccionó mediante zonificación de sitios con mayor concentración de restaurantes como los centros comerciales Ventura Plaza y Unicentro y la avenida el Malecón, principalmente, su desarrollo a través de un trabajo de campo que se ejecutó mediante un previo rutero de visitas previstas en un período de diez días estimados.

\section{Instrumentos}

A título ilustrado, merece la pena destacar que la validez y confiabilidad del instrumento se realizó mediante el criterio y opinión de expertos y especialistas en metodología para la investigación de la Universidad Francisco de Paula Santander, quienes utilizaron un formato preliminar para la revisión según los objetivos de la investigación y en cumplimiento a los principios que deben tenerse en cuenta para evitar errores en el diseño del cuestionario y así lograr un instrumento funcional y estructurado que asegure su validez.

\section{Procedimientos}

La información y datos recolectados se procesaron mediante la categorización de respuestas y tabulación utilizando como herramienta las escalas de medición para una interpretación objetiva de los resultados, además, se elaboró gráficos de cada respuesta para una mejor visualización de la realidad estudiada y facilitar la interpretación y análisis de resultados del cuestionario de tipo cualitativo para la interpretaciones de percepciones de parte de los entrevistados y cuantitativo en la medición de los datos y determinación de promedios ponderados, tendencias y actitudes hacia la oferta y demanda probable de franquicias de parte de los restaurantes de San José de Cúcuta.

Finalmente, el proceso de investigación se desarrolló previo plan de investigación basado en el problema y los objetivos de investigación, lo cual permitió el cumplimiento de las siguientes etapas como identificación del tipo y método de la investigación, determinación y cuantificación de la población objeto de estudio, selección del método de muestreo y cálculo de la muestra, análisis de las técnicas de recolección de la información, validez y confiabilidad del instrumento y determinación de las técnicas para la recolección de información.

\section{4.- Resultados}

La búsqueda y selección de información contenida en fuentes secundarias orientaron la investigación sobre el tema de franquicia y restaurantes, experiencias que aportaron datos significativos al presente trabajo, además, se evidenció que el modelo de franquicia es una atractiva oportunidad para el desarrollo de negocios, que en materia de restaurantes, la franquicia es una estrategia que le permite a los 
establecimientos elevar su nivel competitivo, lo cual se ha evidenciados en las exitosas marcas desarrolladas en Colombia, sin embargo, la capacidad de asociación tiene incidencia en los resultados esperados por los actores involucrados, en este sentido, se debe iniciar procesos de inteligencia comercial como factor clave para identificar ofertas internacionales referentes a restaurantes.

Los resultados de la investigación realizada a la muestra de 87 restaurantes de San José de Cúcuta son un reflejo de toda la población de 877 establecimientos que presentan un determinado desempeño y actitudes hacia la modalidad de franquicias según el siguiente compendio (Tabla 1).

Tabla 1. Síntesis de la Investigación

\begin{tabular}{|c|c|}
\hline Detalles & Resultados \\
\hline Experiencia promedio en el mercado de restaurantes & 7.5 años \\
\hline Capacidad instalada de atención diaria & 61 clientes diarios/restaurante \\
\hline Tipos de comidas que ofrecen & $\begin{array}{l}360 \text { restaurantes de comidas rápidas, } 377 \text { de comida } \\
\text { criolla y } 140 \text { de comida internacional }\end{array}$ \\
\hline Realizan investigación del mercado de consumidores & 254 restaurantes el $29 \%$ \\
\hline Tienen claro el perfil del cliente & 517 restaurantes ó el $59 \%$ \\
\hline Clase social de los clientes que atienden & Alta el $37 \%$ y media el $63 \%$ \\
\hline Miden el nivel de satisfacción del cliente & 132 restaurantes ó el $15 \%$ \\
\hline Nivel de estudios de los clientes & $\begin{array}{l}\text { Secundaria el } 33 \% \text {, superior el } 55 \% \text { y no responden el } \\
12 \%\end{array}$ \\
\hline Medios de comunicación que utilizan & Volantes el $31 \%$, página web el $60 \%$ y prensa el $9 \%$ \\
\hline Marcas identificadas & $\begin{array}{l}\text { Mac Pollo, Hamburguesas del Corral, Brosty, Presto y } \\
\text { Subway }\end{array}$ \\
\hline Valor agregado que ofrecen & $\begin{array}{l}\text { Precios competitivos } 333 \text { restaurantes, calidad } 281 \\
\text { restaurantes, diversidad } 184 \text { restaurantes, y comodidad } \\
79 \text { restaurantes }\end{array}$ \\
\hline Registro de Cámara de Comercio & 482 restaurantes ó el $55 \%$ \\
\hline $\begin{array}{l}\text { Grado de importancia de los factores en un negocio de } \\
\text { comida }\end{array}$ & $\begin{array}{l}\text { Alta importancia rapidez, variedad, trato amable, bajo } \\
\text { precio, parqueo y sazón. } \\
\text { Media importancia buena decoración, servicio a } \\
\text { domicilio y tarjeta de crédito. }\end{array}$ \\
\hline Número de personas vinculadas al restaurante & 5 personas en promedio/ restaurante \\
\hline Promedio de ventas anuales & $\$ 624^{\prime} 828.000$ al año \\
\hline Promedio de activos totales & $\$ 64 ’ 362.000$ \\
\hline Promedio de pasivos totales & $\$ 37^{\prime} 058.000$ \\
\hline Márgen de contribución operativo antes de impuestos & $2.9 \%$ en promedio/restaurante \\
\hline Conocen la modalidad de franquicia & 351 restaurantes \\
\hline Operan bajo la modalidad de franquicia & 53 restaurantes ó el $6 \%$ \\
\hline Ciudad del franquiciador & Bogotá \\
\hline Nivel de satisfacción hacia la franquicia & $\begin{array}{l}\text { Muy alto y ato nivel de satisfacción contrato, marca, } \\
\text { know how, distribución y confidencialidad. } \\
\text { Medio nivel de satisfacción tasa de entrada y regalías. } \\
\text { Bajo nivel de satisfacción la exclusividad. }\end{array}$ \\
\hline Dispuestos a operar bajo franqicia & 272 restaurantes \\
\hline Ofrecería su marca a través de franquicia & 545 restaurantes ó el $62 \%$ \\
\hline
\end{tabular}

Nota: Elaboración propia, 2016. 


\section{5.- Discusión}

Los colombianos a causa de los cambios en los estilos de vida están comiendo con más frecuencia fuera de casa, lo cual a criterio de Blanco y Peralta (2015), obedece al dinamismo que los cambios del entorno laboral ejerce sobre los consumidores, lo que favorece el mercado de comida rápidas, fenómeno que en Cúcuta es una realidad que genera nuevas oportunidades de negocios y como tal debe adaptarse a los requerimientos de esta realidad, adecuando su productos y servicios en función a las necesidades de sus clientes (Sanz, Crissien, García \& Patiño, 2017), lo que debe aprovecharse a través del modelo de franquicia, el cual "representa una de las alternativas más rentables y eficaces para la consecución de objetivos referentes a la cobertura de nuevos mercados, pero también es hoy en día una excelente forma de hacer negocios, ya sea invirtiendo en alguna o adoptándola como estrategia de crecimiento de la empresa (Mosquera, 2010, p.71), por su parte, según la Asociación Colombiana de la Industria Gastronómica (Acodres) como gremio del sector, asegura que en Colombia "se abrieron 5.000 restaurantes aproximadamente para el año 2014 y perciben que hay una tendencia en el reposicionamiento de la comida tradicional y establecimientos especializados en tipo de cocina, como la italiana, árabe, mexicana, francesa, entre otras" (OCEX, 2016, p.12).

Dentro de este marco, es importante señalar que para el caso de Cúcuta, se han establecido desde hace una década empresas de comidas rápidas como McDonald's, Subway, Hamburguesas El Corral, Mimo’s, Helados San Jerónimo, Juan Valdés y Mc Pollo, entre otras.

Los resultados de la investigación aplicada a la población de 877 restaurantes señalan la existencia de un prospecto de mercado para franquicias de restaurantes y comidas rápidas en Cúcuta y su Área Metropolitana dado que un $31 \%$ de los restaurantes analizados están dispuestos a operar en franquicia, para ello se recomienda que el franquiciado seleccione la marca a través de análisis previos, dado que es el elemento de diferenciación que permitirá al nuevo negocio alcanzar una notoriedad en el mercado frente a las otras marcas competidoras; Prieto y Villa (2011), adicionalmente, los franquiciadores para desarrollarse en mercados de interés están obligados a emprender acciones estratégicas orientadas al fortalecimiento de buenas relaciones con los franquiciados y lograr así una posición consolidada en el mercado; aspectos que se constituyen en temas para investigaciones futuras relacionadas con planes de mercadeo para el posicionamiento de una marca en franquicia

\section{6.- Conclusiones}

La investigación documental dentro de una perspectiva deductiva permitió a la investigadora entender que el modelo de franquicias para restaurantes y comidas rápidas en San José de Cúcuta es una oportunidad de negocios dado los prospectos de mercado que se evidencian en experiencias anteriores, además, con este modelo según esos antecedentes, las empresas logran un alto nivel competitivo. 
El modelo de franquicia implica una efectiva relación comercial entre franquiciador y franquiciado mediante políticas y condiciones claras que deben protocolizarse en un Contrato de franquicia. Los cambios en los estilos de vida, el crecimiento de la población, los horarios laborales y la mayor participación de la mujer en el mercado laboral, entre otros; son factores que están incidiendo en la demanda de comidas rápidas y restaurantes en general, fenómeno en que Cúcuta no es una excepción.

Como estrategia de expansión empresarial, las franquicias generan mayor productividad y competitividad, menores riesgos, rápido posicionamiento, identidad corporativa y responsabilidad compartida, por lo tanto para el caso de restaurantes y comidas rápidas ,constituyen atractivos prospectos de negocios,.

El Contrato de franquicia debe señalar los deberes y derechos de parte delfranquiciador y franquiciado, resaltando en especial el valor inicial de la franquicia y las regalías involucradas según resultados comerciales,

Participar en un negocio en calidad de franquicia obliga a los interesados a realizar previas investigaciones del mercado de interés a fin de determinar el desempeño de los restaurantes de San José de Cúcuta y sus actitudes hacia la modalidad de franquicias y establecer los gustos y preferencias de parte de la población hacia el consumo de comidas rápidas, al respecto, al respecto, los resultados de la investigación señalan la existencia de un prospecto de mercado para franquicias de restaurantes y comidas rápidas en Cúcuta y su Área Metropolitana.

\section{7.-Referencias}

Ariza, A. B., \& Miranda, P. P. (2015). Competitividad y estructura organizacional de las PYMES del sector alimento en Barranquilla. Una perspectiva conceptual. Desarrollo gerencial, 7(2). Pp. 170187. http://dx.doi.org/10.17081/dege.7.2.1187.

Barriga Monroy, M. (2011). Estado del arte y definición de términos sobre el tema "La investigación en educación artística" El Artista, núm. 8, Universidad Distrital Francisco José de Caldas Pamplona, Colombia, pp. 224-241.

Cámara de Comercio de Cúcuta (2013). Observatorio Económico, Mercado Laboral.

Colfranquicia (2014). Feria Fanyf: El punto de encuentro efectivo para encontrar la franquicia y el negocio de sus sueños, Bogotá.

Delgado, D. (2009). Análisis Socio Económico y de Mercado del Sector de Comidas Rápidas en San José de Cúcuta, Administración de Empresas, UFPS. 
Del Vecchio, D. S., Borrero, T. C., Guiliany, J. G., \& Villanueva, M. P. (2017). Marketing Educativo Como Estrategia de Negocio en Universidades Privadas. En Desarrollo Gerencial, 9(1), 160-175.

Durán, S., Crissien, E., Virviesca, J. y García, J. (2017). Estrategias gerenciales para la formación de equipos de trabajos en empresas constructoras del Caribe colombiano. Revista Espacios, vol. 38. Nro. 13. http://www.revistaespacios.com/a17v38n13/17381324.html.

Durán, S., Fuenmayor, A., Cárdenas, S. \& Hernández, R. (2016). Emprendimiento como proceso de responsabilidad social en instituciones de educación superior en Colombia y Venezuela. En Desarrollo Gerencial. Universidad Simón Bolívar-Colombia, 8(2), 58 - 75.

Escalante, Z., \& García, J. (2010). Modelos de Negocios en Entornos de Desarrollo Tecnológico. CICAG, 6(1), 77-104.\}.

Estudio sobre el uso del Sistema de Franquicia, (s.f.), España, p.1-62 recuperado de http://www.procesa.es/attachments/article/83/F.

García, J., Durán, S., Parra, M. y Márceles, V. (2017). Dirección estratégica del talento humano para el fomento de valores en los cuerpos policiales venezolanos. Revista Espacios, volumen 38, nro. 32, $16-28$.

García-Guiliany, J. E. Duran, S. E., \& Ronald, Prieto-Pulido. (2017). Políticas de gestión de talento humano para el desarrollo de competencias gerenciales en empresas metalmecánica. FACE: Revista de la Facultad de Ciencias Económicas y Empresariales, 17(2), 130-141.

Gómez, O. (2012). Costo, volumen, precio y utilidad: dinámica del desempeño financiero. Industria Confecciones Infantiles, Artículo de Investigación Científica y Tecnológica, Universidad Santo Tomás, Bucaramanga, p.55-65.

Herrera, M. F. N., \& Moreno, A. M. M. (2009). El contrato de franquicia: aportes y tendencias en el derecho comparado sobre la responsabilidad del franquiciador. Vniversitas, (119), 279-304. Pontificia Universidad Javeriana Bogotá, Colombia

Jany, J. (2000). Investigación Integral de Mercados, Bogotá DC, Editorial McGraw Hill, p.47-375.

Llain-Arenilla, S. e Insignares, S. (2016). Efectos del Tratado de Libre Comercio entre Colombia y Estados Unidos en torno al contrato de franquicia internacional, Vniversitas, núm. 132, pp. 21-57, Pontificia Universidad Javeriana, Bogotá, Colombia.

Méndez, C. (2007). Metodología, Diseño y Desarrollo del Proceso de Investigación con Énfasis en Ciencias Empresariales. Bogotá D.C: Limusa Noriega Editores. 
Mosquera, F. (2010). La Franquicia Una Estrategia De Crecimiento Empresarial, Revista MBA EAFIT, Medellín.

Órganos de Control Externo Autonómicos (OCEX) (2016). Franquicias Gastronómicas, Perfil de Servicio, en colaboración con el Ministerio de Comercio Exterior y Turismo del Perú, Bogotá D.C.

Prieto, R., \& Villa, C. (2011). Estrategias de mercadeo interno para optimizar la calidad de servicio en empresas ferreteras. Revista Innovación y Gerencia, 4(2), 84-100.

Prieto, R., Burgos, C., García, J., \& Rincón, Y. (2016). Mercadeo interno para optimizar la calidad de servicio en la banca universal. Revista venezolana de gerencia, 21(73). P.p. 102-119.

Promperú (2004). Perfil del Mercado de Franquicias en Colombia, Programa de Promoción de Exportación de Servicios, La franquicia en Colombia un negocio en auge, Bogotá, p.1-85

Quiroga Olaya, A. (2012). Contrato de franquicia: propuesta de regulación en la Comunidad Andina, en Revista de Derecho Privado, 48, 1-31.

Ramírez González, A. (1996). Metodología de la Investigación CientíficaPontificia Universidad Javeriana .Facultad de Estudios Ambientales y Rurales, Bogotá, p.1-111

Riaño, V. (2013). El Caso de Perdurabilidad Empresarial de Crepes \& Waffles desde una Perspectiva de Responsabilidad Social Empresarial e Innovación en Valor en Colombia Universidad del Rosario Escuela de Administración Maestría en Dirección de Empresas Bogotá D.C.

Revista Semana (2012). El mercado de comidas rápidas: un negocio sabroso. Recuperado de:http://www.semana.com/economia/articulo/el-mercado-comidas-rapidas-negociosabroso/265173-3

Rodríguez, M. \& Mora, N. (2012). K-LISTO: Franquicia como una estrategia de expansión Universidad Colegio Mayor De Nuestra Señora Del Rosario Facultad de Administración

Schlesinger, M. (2008). Percepción del posicionamiento estratégico en el ámbito de las franquicias de comida rápida en España, Facultad de Ciencias Económicas y Sociales de la Universidad del Zulia. Venezuela, p.1-165

Universidad Pedagógica Experimental Libertador (2002). Manual de Trabajos de Grado de Especialización y Maestría y Tesis Doctorales, Caracas, p.3-69

Uribe Merino, J. (2006). Las prácticas alimentarias relacionadas con la búsqueda del ideal corporal. El caso de la ciudad de Medellín (Colombia). En Boletín de Antropología Universidad de Antioquia, 20, (37), 227-250. 


\section{Ytron T U N I V E R S I D A D

Vílchez. Gil, A., (2003), Gestión de inventario. Relación con los proveedores en franquicias de comida rápida. En Revista Venezolana de Gerencia, 8, (23), 510-525. 